\title{
Access to Care for Multiple Sclerosis in Times of Economic Crisis in Greece - the HOPE II Study
}

\author{
Kyriakos Souliotis ${ }^{1,2^{*}}$, Elena Alexopoulou ${ }^{3,4}$, Manto Papageorgiou ${ }^{3}$, Anastasia Politi ${ }^{5}$, Panagiota Litsa ${ }^{2}$, \\ Xenophon Contiades ${ }^{3}$
}

\begin{abstract}
Background: While there is currently no cure for multiple sclerosis (MS), treatment with biologic diseasemodifying drugs (bDMDs) can reduce the impact of the condition on the lives of patients. In Greece, the regulatory change in the distribution system of bDMDs, limited their administration through the designated pharmacies of the National Organization for Healthcare Services Provision (EOPYY) or the National Health System (ESY) hospitals, thus potentially impacting access to MS treatment. In this context, the aim of this paper was to assess the barriers to bDMDs, by recording MS patients' experiences.

Methods: A survey research was conducted between January and February 2014 in Athens and 5 other major Greek cities with the methods of personal and telephone interview. A structured questionnaire was used to elicit socio-economic and medical information, information related to obstacles in accessing bDMDs and medical treatment, from MS patients that visited EOPYY pharmacies during the study period.

Results: During the last year $69 \%$ of 179 participants reported that the distribution system of bDMDs has improved. Thirteen percent of participants encountered problems in accessing their medication, and $16.9 \%$ of participants in accessing their physician, with the obstacles being more pronounced for non-Athens residents. Frequent obstacles to bDMDs were the distance from EOPYY pharmacies and difficulties in obtaining a diagnosis from an EOPYY/ESY physician, while obstacles to medical care were delays in appointment booking and travel difficulties.

Conclusion: Even though the major weaknesses of the distribution system of bDMDs have improved, further amelioration of the system could be achieved through the home delivery of medicines to patients living in remote areas, and through the development of a national MS registry.

Keywords: Multiple Sclerosis (MS), Patient Access, Biologics, Pharmaceutical Policy, Recession, Greece

Copyright: (C) 2016 by Kerman University of Medical Sciences

Citation: Souliotis K, Alexopoulou E, Papageorgiou M, Politi A, Litsa P, Contiades X. Access to care for multiple sclerosis in times of economic crisis in Greece - the HOPE II study. Int J Health Policy Manag. 2016;5(2):83-89. doi:10.15171/ijhpm.2015.173
\end{abstract}

\author{
Article History: \\ Received: 12 April 2015 \\ Accepted: 23 September 2015 \\ ePublished: 27 September 2015
}

\section{Key Messages}

Implications for policy makers

- Distribution of biologic disease-modifying drugs (bDMDs) for multiple sclerosis (MS) through the National Organization for Healthcare Services Provision (EOPYY) pharmacies has improved during the last year.

- Despite the improvement in the operation of the distribution system, one in 8 patients continues to encounter barriers in accessing MS medication.

- There are inequalities in accessing care, with patients living outside Attica encountering more obstacles compared to Attica residents.

- A potential solution in tackling the existing inequalities in accessing bDMDs is the home delivery of medicines to MS patients living in remote areas with poor means of transportation.

- The development of a national MS registry could further facilitate the distribution process of bDMDs, ensuring patient eligibility and thus enhancing access to care and the health status of MS patients.

Implications for public

The present study has been conducted in an effort to highlight the obstacles in accessing treatment for multiple sclerosis (MS) with biologic disease-modifying drugs (bDMDs) in Greece in the era of economic crisis. The economic downturn and the need for fiscal consolidation have led to the implementation of a number of cost-containment measures in the Greek pharmaceutical market, such as the recent change in the distribution system of costly medicine, including bDMDs for MS. Thus, during the last two years, Greek MS patients have abruptly moved from a status of unrestricted and fairly easy access to bDMDs to a complex distribution system which has limited their access to effective treatment. In this context, the purpose of this study is to increase awareness regarding the obstacles encountered by Greek MS patients in accessing their treatment, and to propose measures that could potentially improve the situation. 


\section{Background}

The dimensions of quality in health according to Maxwell ${ }^{1}$ are accessibility, equality, responsiveness to the needs of patients and the community, social acceptance, efficiency, and effectiveness. People who suffer from chronic diseases such as multiple sclerosis (MS) use health services more frequently than the general population. This brings patients face to face with the potential weaknesses of the health system that affect quality of healthcare, impeding accessibility as well as the smooth operation of these services. ${ }^{2,3}$

Patients with MS need to have direct access to pharmaceutical treatment that best meets their needs. ${ }^{4}$ While there is currently no cure available for MS, there are pharmaceutical interventions, ie, biologic disease-modifying drugs (bDMDs, eg, beta interferon-1a, beta interferon-1b, glatiramer acetate, natalizumab, fingolimod, mitoxantrone), that can significantly reduce the impact of the condition on the lives of patients, by treating relapses and stabilizing the health status of patients, ${ }^{4-6}$ thus improving their quality of life and leading to reduced loss of productivity, and reduced direct and indirect costs. In this respect, in order to assess the level of access to effective MS treatment, the accessibility of patients to bDMDs is usually evaluated by the academic community. ${ }^{7-9}$

In Greece, MS treatment with bDMDs has been traditionally fully reimbursed by social insurance according to legislation, while according to the 2013 MS Barometer report, approximately $70 \%$ of Greek MS patients are being treated with bDMDs, which is one of the highest percentages in Europe. ${ }^{9}$ In the context of Greece's economic adjustment programme, a number of policy measures and regulatory changes have been implemented in the Greek pharmaceutical sector since 2010, in an effort to rationalize public expenditure and control excess prescribing. Thus, the latest regulatory change in the distribution system of high-cost, fully reimbursed, medicines (ie, medicines defined by Law $3816 / 2010$ ), including bDMDs for MS, has limited access to high-cost pharmaceuticals, by allowing their administration only through the publicly-owned, designated pharmacies of the National Organization for Healthcare Provision (EOPYY) or the National Health System (ESY) hospitals (medicines administered by hospitals if: $(a)$ the medicine is administered by intravenous infusion and therefore is only for hospital use, or $(b)$ the use of the medicine must start under hospital monitoring and can be continued outside the hospital but under hospital surveillance, according to its packaging information -"blue box"), and only when their prescription is accompanied by an appropriate medical diagnosis issued by a hospital physician. The aim of this measure was to contain public pharmaceutical costs and to ensure that high-cost medicines such as bDMDs are prescribed according to official treatment guidelines and protocols. ${ }^{10}$

In the light of this regulatory change, a recent study by Souliotis et $\mathrm{al}^{11}{ }^{11}$ ie, HOPE I (Health Outcomes Patient Environment I) study, assessed access to high-cost medicine for the treatment for rheumatoid arthritis (disease-modifying antirheumatic drugs, DMARDs) in Greece, concluding that patients are facing increased barriers to access timely and effective treatment under the new distribution system. In this context, the objective of the present study, ie, HOPE II, was to assess the operation of the high-cost medicine distribution system one year later, and in another disease area, ie, in MS, based on the experiences of patients. Specifically, this study aims to assess the access of MS patients primarily to bDMDs and secondarily to medical care following the implementation of the new distribution system, drawing on the different obstacles presented to patients and on their correlation to the socio-economic characteristics of patients. Even though the main objective of the paper was to evaluate the access to bDMDs for MS patients, data on access to MS medical care was also collected in order to identify potential correlations between the obstacles encountered in the 2 types of care. MS patients need to see their doctor in order to get a prescription for a bDMD, therefore the frequency and the different obstacles related to medical visits may influence access to bDMDs. Moreover, by collecting data on both medical and pharmaceutical MS treatment, we attempted to review access to MS care as a whole in the era of economic crisis in Greece.

\section{Methods}

For the HOPE II study a survey research was conducted between January and February 2014 in Athens (Attica prefecture) and other urban areas in Greece. Data for the study were collected with personal and telephone interviews by filling out a questionnaire, in 7 pharmacies of EOPYY, located in Athens ( 2 pharmacies) and other major cities of the Greek mainland (4 pharmacies in Ioannina, Lamia, Thessaloniki, Alexandroupoli) and the islands (1 pharmacy in Herakleion, Crete). The target population was defined as the finite population subset corresponding to those MS patients who, during the study period, visited EOPYY pharmacies in order to obtain their MS pharmaceutical prescription. Patients with 12 months of treatment at least before the starting time of the survey were included in the sample.

The purpose of the questionnaire was to examine MS patients' attitude towards the conditions of medical and pharmaceutical care for their illness. The questionnaire was formulated in such a way as to safeguard the anonymity of respondents. The respondents were kindly requested to respond to all questions with the utmost accuracy. The questionnaire consisted of 4 tiers of questions including multiple-choice or open-ended questions to collect information on: (a) Demographic and financial characteristics of MS patients, ie, age, gender, place of birth, place of residence, education level, level of monthly income, source of income, type of profession, selfassessment of economic situation, type of health insurance; (b) Clinical and medical parameters related to the history and management of MS, ie, year of initial diagnosis of disease, type of treating physician, frequency of medical visits, mode of administration of treatment; (c) The frequency and type of difficulties/problems experienced by the participants in accessing pharmaceutical and medical MS care during the last year (eg, "Have you encountered obstacles in accessing MS pharmaceutical care during the last 12 months?," "If yes, this is due to: $a$. Distance from EOPYY pharmacies, $b$. Difficulties in prescription process due to limited accessibility to physician's clinic, $c$. Difficulties in obtaining the required medical diagnosis by a hospital physician" etc.) and (d) The patients' opinion about the quality of pharmaceutical care provision with regards to the distribution of MS medication through the EOPYY pharmacies, measured on a 5-point Likert 
scale (ie, "Overall, do you think that the process of obtaining your MS medication through the EOPYY pharmacies has improved during the last 12 months?"). In the 2 multiplechoice questions regarding the type of obstacles experienced in accessing pharmaceutical and medical care, participants could provide more than one answer, ie, "You may choose more than one obstacles."

The prevalence of MS in Greece is estimated at 10000-12 000 based on data from the Greek MS Patient Association. For the purposes of the study and in accordance with its cost and time limitations, a sample of $2 \%$ of the population was targeted, ie, approximately 200 questionnaires. Because of the selected method of personal interview, a high response rate was reached, namely, 180 out of the 200 questionnaires. From these questionnaires, 179 fully completed questionnaires were suitable for statistical processing. Statistical analysis included the calculation of empirical frequency and relative frequency distributions. Population parameters were estimated using measures of location (arithmetic mean) and dispersion (standard deviation). Differences between populations were tested with $t$ tests. The level of statistical significance was set at $1 \%$.

\section{Results}

The study population consisted of 179 MS patients who visited one of the participating EOPYY pharmacies, either in Athens $(51 \%, \mathrm{n}=91)$ or in other 5 major Greek cities $(49 \%$, $\mathrm{n}=88$, ie, Herakleion, $\mathrm{n}=25$; Ioannina, $\mathrm{n}=23$; Lamia, $\mathrm{n}=18$; Thessaloniki, $n=13$; Alexandroupoli, $n=9$ ) during the study period. The majority of the study participants belonged to the $36-50$ age-group, while $63 \%$ of them were women and $37 \%$ were men, with a mean time since MS diagnosis of 8.4 years (standard deviation $[S D]=6.4)$ and 7.7 years $(S D=5.0)$ respectively. Most patients had received secondary education and reported a monthly income of up to $€ 1000$, while $92 \%$ of patients did not have private insurance. The detailed demographic and socio-economic characteristics of the patient population are presented in Table 1.

\section{Access to Multiple Sclerosis Medical Treatment}

Access to MS medical treatment was assessed through the study questionnaire in order to identify potential factors/ obstacles that could further impact on access to bDMDs for MS patients, and to assess access to MS care as a whole.

Based on the study results, $60.2 \%$ of MS patients reported visiting their treating physician once every three months, while $16.5 \%$ of patients visited their doctor once a month over the previous year (Figure 1). Most patients (81\%) preferred visiting physicians at ESY hospitals, while the rest opted for private physicians (contracted or non-contracted with EOPYY), or physicians at EOPYY health centers. During their physician visit, $88 \%$ of patients were prescribed MS medication which did not require short-term hospitalization. One in 6 respondents, ie, $16.9 \%$ of all patients, answered positively to the question "Have you encountered obstacles in accessing MS medical care during the last 12 months?," while this percentage was higher among those who were prescribed medication that did not require short-term hospitalization, ie, $17.7 \%$ (versus $9.5 \%$ among patients whose medication require short-term hospitalization). The most frequently reported
Table 1. Patient Characteristics

\begin{tabular}{|c|c|c|}
\hline $\begin{array}{l}\text { Demographic, and Socio-economic } \\
\text { Characteristics }\end{array}$ & No. of Patients & $\%$ \\
\hline Total & 179 & 100.0 \\
\hline \multicolumn{3}{|l|}{ Gender } \\
\hline Men & 67 & 37.3 \\
\hline Women & 112 & 62.7 \\
\hline \multicolumn{3}{|l|}{ Age (years) } \\
\hline$\leq 25$ & 10 & 5.7 \\
\hline $26-35$ & 37 & 20.3 \\
\hline $36-50$ & 95 & 53.1 \\
\hline $51-65$ & 34 & 19.2 \\
\hline$\geq 66$ & 3 & 1.7 \\
\hline \multicolumn{3}{|l|}{ Education } \\
\hline Compulsory education & 22 & 12.1 \\
\hline Secondary education & 80 & 44.5 \\
\hline Technological education & 21 & 11.6 \\
\hline Higher education & 44 & 24.9 \\
\hline Postgraduate education & 12 & 6.9 \\
\hline \multicolumn{3}{|l|}{ Monthly income } \\
\hline$\leq € 500$ & 33 & 18.1 \\
\hline$€ 501-€ 1000$ & 86 & 48.1 \\
\hline$€ 1001-€ 1500$ & 50 & 28.1 \\
\hline$€ 1501-€ 2000$ & 2 & 1.3 \\
\hline$€ 2001-€ 2500$ & 5 & 2.5 \\
\hline$>€ 2500$ & 3 & 1.9 \\
\hline \multicolumn{3}{|l|}{ Self-assessment of economic status } \\
\hline Very good & 2 & 1.3 \\
\hline Good & 24 & 13.3 \\
\hline Fair & 87 & 48.7 \\
\hline Bad & 53 & 29.7 \\
\hline Very bad & 13 & 7.0 \\
\hline \multicolumn{3}{|l|}{ Private insurance } \\
\hline Yes & 15 & 8.3 \\
\hline No & 164 & 91.7 \\
\hline \multicolumn{3}{|l|}{ Place of residence } \\
\hline Prefecture of Attica & 88 & 49.2 \\
\hline Outside of prefecture of Attica & 91 & 50.8 \\
\hline
\end{tabular}

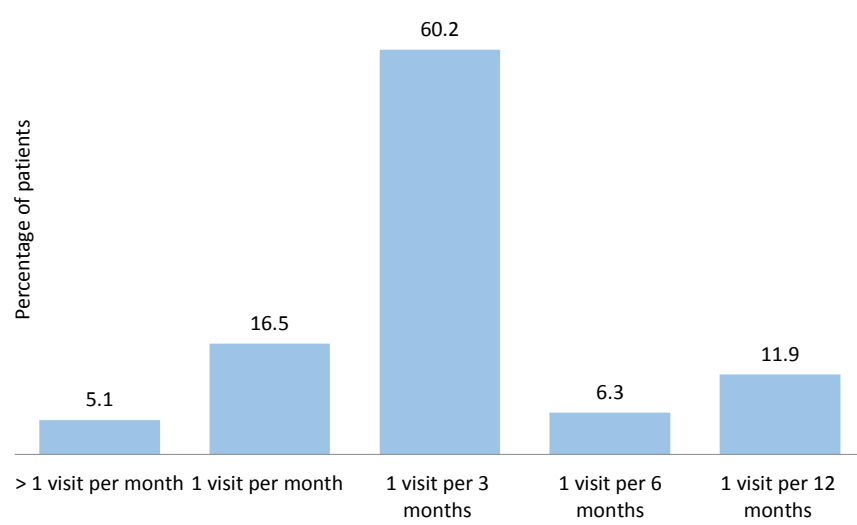

Figure 1. Distribution of Patients According to the Frequency of Physicians' Visits. 
cause of reduced accessibility to the MS physician was the delay in scheduling an appointment with the doctor, followed by travel difficulties and the inability to pay a non-contracted private physician (Table 2 ).

According to the analysis of the study results, the level of monthly income was shown to be associated with the frequency of scheduled medical visits, as the percentage of patients delaying their medical visit appeared to be higher among those with a monthly income less than $€ 500$, ie, $13.8 \%$, compared to the respective percentage reported among patients with an income higher than $€ 500$, ie, $7 \%$, yet their difference was not significant $(P=.3)$. Low-income $(\leq € 500)$ MS patients also reported relatively high inability to pay for a non-contracted private physician $(10.4 \%$ versus $3.1 \%$; $P=.2)$, as well as more difficulties due to the distance from the physician's clinic ( $3.5 \%$ versus $1.6 \% ; P=.5)$. As further analysis of the distribution of patients with respect to their income and place of residence showed that the percentage of Athens residents with a monthly income less than $€ 500$ was $5.8 \%$, compared to the significantly higher respective percentage of $32.4 \%(P<.001)$ among non-Athens residents, we can deduce that patients residing outside Athens have a higher risk of experiencing a barrier to access their MS treatment.

\section{Access to Multiple Sclerosis Pharmaceutical Treatment}

With regards to accessing MS treatment with bDMDs, approximately one in eight respondents, ie, $13.3 \%$ of all patients, answered positively to the question "Have you encountered obstacles in accessing MS pharmaceutical care during the last 12 months?", while, again, this percentage was higher among those who were prescribed medication that did not require short-term hospitalization, ie, $14 \%$ versus (the nonstatistically lower) $4.8 \%$ among patients whose medication require short-term hospitalization $(P=.1)$. In this respect, the reported barriers were related to the distribution channels of MS medication, and also to the prescribing process. In particular, the most frequently reported obstacle encountered in accessing bDMDs was the long distance required to reach an EOPYY pharmacy, followed by the reduced accessibility of patients to their treating physician, and the difficulties

Table 2. Commonly Reported Barriers to Accessing MS Medical Treatment

\begin{tabular}{lll}
\hline Commonly Reported Obstacles & No. of Cases & $\%$ \\
\hline $\begin{array}{l}\text { Delay in scheduling an appointment with a } \\
\text { physician working at an NHS hospital or at }\end{array}$ & 15 & 40.6 \\
$\begin{array}{l}\text { EOPYY's health centers } \\
\begin{array}{l}\text { Difficulties of travel to and from the clinic due to } \\
\text { health reasons or lack of means of transportation }\end{array}\end{array}$ & 9 & 24.3 \\
$\begin{array}{l}\text { Inability to pay a non-contracted private } \\
\text { physician }\end{array}$ & 8 & 21.6 \\
$\begin{array}{l}\text { Distance from physician's clinic } \\
\begin{array}{l}\text { Difficulty in scheduling an appointment with a } \\
\text { contracted private physician }\end{array}\end{array}$ & 1 & 10.8 \\
\hline Total & 37 & 2.7 \\
\hline
\end{tabular}

Abbreviations: MS, multiple sclerosis; NHS, National Health Service; EOPYY, National Organization for Healthcare Services Provision.

Note: This table presents the frequency and relative frequency distribution of barriers experienced by patients who gave a positive answer to the question: "Have you experienced obstacles in accessing MS medical care during the last 12 months?" in obtaining the required medical diagnosis by a hospital physician (Table 3).

The barriers to accessing bDMDs were shown to be statistically significantly more pronounced for non-Attica residents who live in urban centers of the Greek mainland, as the mean number of obstacles reported by them was 2.3 compared to 0.6 for Attica residents $(P=.0)$.

Almost $98 \%$ of MS patients who experienced obstacles in accessing their medication, were eventually able to receive fully reimbursed bDMDs through the designated distribution channels (ie, EOPYY pharmacies or ESY hospitals), and therefore did not have to either pay-out-of-pocket or make any changes in their therapeutic regimen (eg, did not have to switch to another - non-bDMD - MS medicine that is available in retail pharmacies). In addition, $9 \%$ of these patients missed or delayed treatment, which resulted in a deteriorated health status for $5 \%$ of them.

Moreover, with regards to the attitude of patients towards the distribution of MS medication through the EOPYY pharmacies, the study participants were asked their personal opinion on whether the distribution process has improved during the last twelve months. Thus, $69 \%$ of MS patients answered that the distribution process has indeed improved or improved a lot (Figure 2), while this percentage was higher among non-Attica residents, ie, 71\% (versus $66.7 \%$ among Attica residents; $P=.5$ ).

Further analysis of the data by geographic region and by type of administration of treatment (medication requiring shortterm hospitalization or no hospitalization) showed that the process improved or substantially improved particularly outside Attica, and especially among patients who receive treatment that does not require short-term hospitalization (73.6\%) (Figure 3). These patients need to access EOPYY

Table 3. Commonly Reported Barriers to Accessing MS Pharmaceutical Treatment

\begin{tabular}{lcc}
\hline Commonly Reported Obstacles & No. of Cases & $\%$ \\
\hline Distance from EOPYY pharmacies & 9 & 28 \\
$\begin{array}{l}\text { Difficulties in prescription process due to limited } \\
\text { accessibility to physician's clinic }\end{array}$ & 5 & 15 \\
$\begin{array}{l}\text { Difficulties in obtaining the required medical } \\
\text { diagnosis by a NHS hospital physician }\end{array}$ & 5 & 15 \\
$\begin{array}{l}\text { Difficulties related to the availability or the } \\
\text { administration of MS medication in ESY hospitals }\end{array}$ & 3 & 9 \\
$\begin{array}{l}\text { Non-availability of medication in ESY hospitals } \\
\text { Distance from ESY hospitals }\end{array}$ & 3 & 9 \\
\hline $\begin{array}{l}\text { Distance from private pharmacies } \\
\text { Pharmacists' strikes }\end{array}$ & 2 & 6 \\
\hline $\begin{array}{l}\text { Difficulties in the administration/non-availability } \\
\text { of medication in private pharmacies }\end{array}$ & 1 & 6 \\
$\begin{array}{l}\text { Difficulties in the administration/non-availability } \\
\text { of medication in EOPYY pharmacies }\end{array}$ & 1 & 3 \\
\hline Total & 33 & 100 \\
\hline
\end{tabular}

Abbreviations: MS, multiple sclerosis; NHS, National Health Service; EOPYY, National Organization for Healthcare Services Provision; ESY, National Health System.

Note: This table presents the frequency and relative frequency distribution of barriers experienced by patients who gave a positive answer to the question: "Have you experienced obstacles in accessing MS medical care during the last 12 months?" 


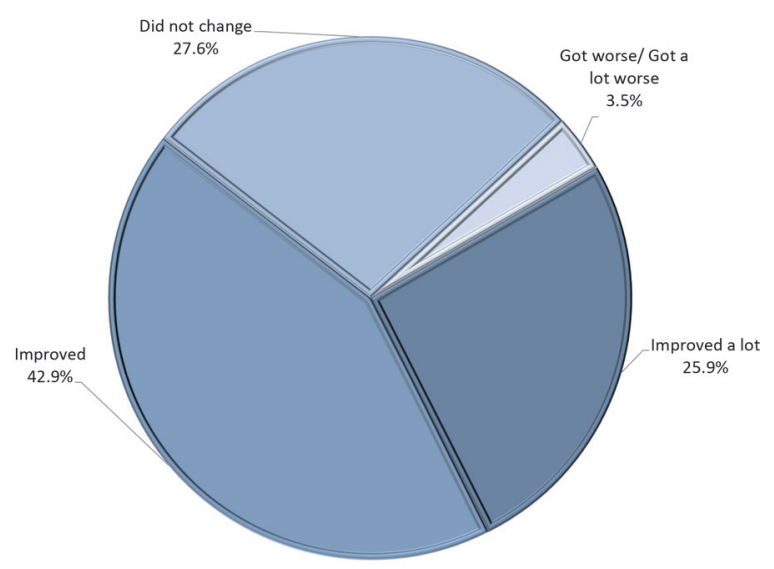

Figure 2. Patients' Answer to the Question: "Overall, Do You Think That the Process of Obtaining Your MS Medication Through the EOPYY Pharmacies Has Improved During the Last 12 Months?"

pharmacies to get their medication, as compared to patients who receive treatment that requires hospitalization, in which case the treatment is provided by the hospital.

\section{Discussion}

A number of studies have shown that there is wide and inconsistent variation in the access of MS patients to bDMDs across Europe, showing that there are still significant inequalities in access to effective MS care. ${ }^{6-8}$ Kobelt and Kasteng ${ }^{6}$ estimated that the proportion of MS patients on DMDs varied between approximately 6\% and 83\% among European countries. Wilsdon et $\mathrm{al}^{7}$ found a range of $13 \%$ and $69 \%$ in a more recent study, while the 2013 MS Barometer reported a range of $11 \%$ and $70 \% .^{8}$

A number of factors may explain the differences in the use of bDMDs, but the most important determinants of access to bDMD treatment are, on one hand, issues related to their reimbursement process and differences in affordability across European economies, and on the other hand issues related to medical practice, ie, differences pertaining the diagnosis and management process of the disease. ${ }^{4,6,7}$

In Greece, even though the overall score for access to treatment and therapies in general is below the European average (especially in terms of psychological care and rehabilitation services), access to bDMDs has been particularly high in the past years. ${ }^{8}$ Nevertheless, during the Greek economic crisis, access to fully reimbursed, high-cost medicine for serious, costly illnesses such as, rheumatoid arthritis (RA) and MS has been restricted, by distributing these medicines exclusively through the pharmacies of EOPYY or the pharmacies of ESY hospitals, and not through private pharmacies. This measure was implemented primarily to reduce public spending for high-cost medicines, as in this way, the State purchased them at discounted, hospital price, without incurring the extra cost resulting from the reimbursement of medicines at pharmacy, retail price. In addition, the measure aimed at a higher control of the eligibility of patients and the compliance to clinical guidelines, by applying additional requirements in the distribution process, ie, a medical diagnosis issued by an EOPYY/ESY doctor that needs to be submitted together with the prescription, in order to obtain the medicine.

However, the implementation of the new distribution system did not come without problems. The complexity of the system stemming from the increased administrative barriers and the limited points of distribution, combined with the medicine shortages that occurred in ESY hospitals due to the instability of the Greek pharmaceutical market during the first months of the system's operation, severely affected access to medicines, especially for patients residing outside the prefecture of Attica. This resulted in anxiety, confusion and discomfort for patients, who often had to travel long distances to ensure access to their medicines. As shown in the HOPE I study by Souliotis et al, ${ }^{11}$ one in 2 patients with RA reported problems in accessing biologic DMARDs during 2012 through the new system, which they attributed to the long distance from EOPYY pharmacies, difficulties in accessing a prescribing

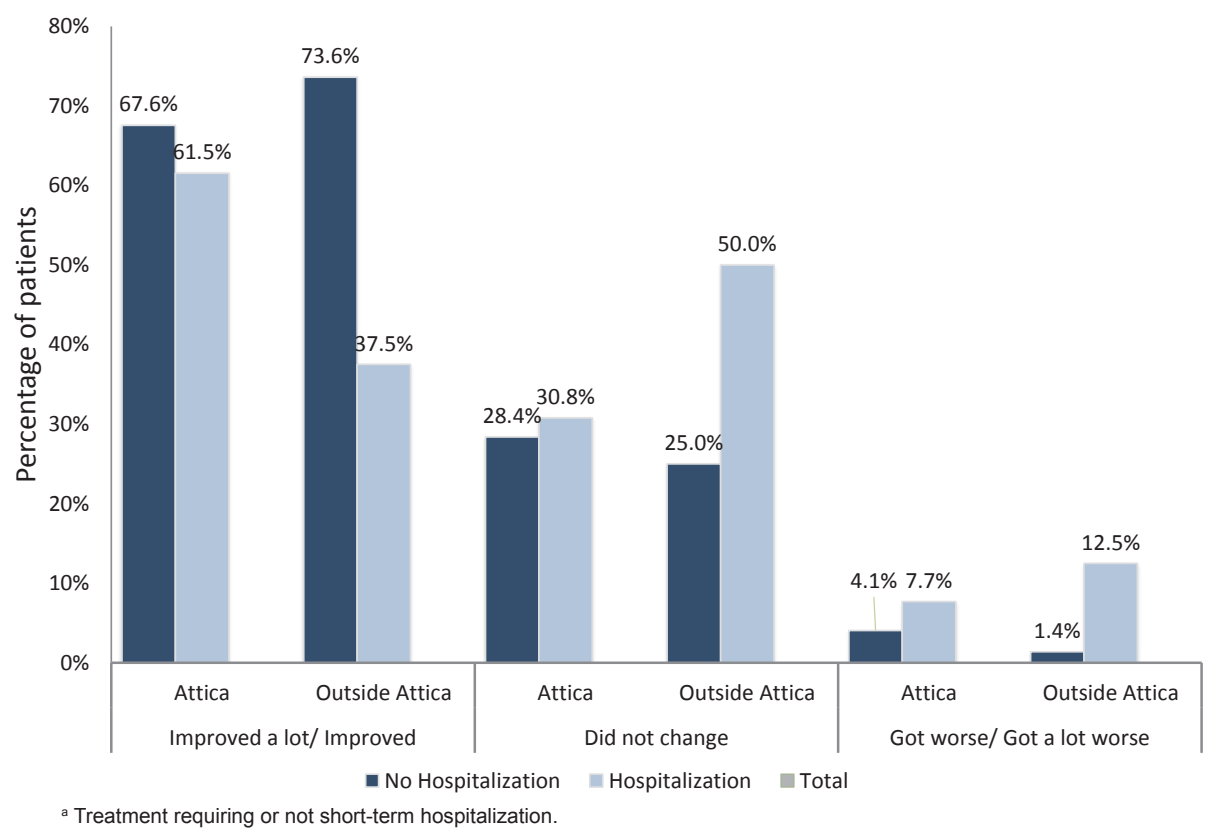

Figure 3. Patients Attitude Towards the Improvement of the Distribution System of MS medication, during the Last 12 Months, by Place of Residence and Type of Pharmaceutical Treatmenta. 
doctor and to the non-availability or difficulty of finding their medication at an ESY hospital or EOPYY pharmacy.

The present, HOPE II study, which was conducted one year after the HOPE I study and in a different disease area, showed that according to the majority of respondents, there has been improvement and streamlining in the operation of the distribution system. This is also confirmed by the fact that, as mentioned before, $9 \%$ of the study population reported delaying or missing treatment which subsequently led to the deterioration of health status for $5 \%$ of them. These percentages are substantially lower compared to the corresponding percentages reported in HOPE $\mathrm{I}^{11}$ one year earlier where $18 \%$ of patients with RA reported delay or loss of treatment, leading to the deterioration of health status for $22 \%$ of them. The improvement in accessing high-cost medicine can be attributed to the operation of additional points of administration in the distribution system, ie, additional EOPYY pharmacies, especially outside Attica as well as to the relative stabilization of the Greek pharmaceutical market, during the last year.

At this point, it should be stressed, however, that the two studies looked at different patient populations in different points in time, and therefore differences in results could also be related to the differences in clinical practice and prescribing habits of attending physicians in Greece. Based on the literature, overall uptake of bDMDs for MS is higher in Europe, compared to the uptake of DMARDs for RA, despite the fact that the effectiveness of biologics in the latter disease area is well-documented. ${ }^{7}$ This could be because the prevalence of MS is lower compared to that of RA, and therefore the budget impact of the prescription of bDMDs among MS patients is more limited compared to that of the prescription of DMARDs among RA patients, thus resulting in better, less restricted access to innovative medications for MS patients on affordability grounds., ${ }^{72-15}$

Yet, despite the improvement in the operation of the distribution system, problems still exist, with one in eight MS patients reporting barriers in accessing bDMDs. Similarly to the previous HOPE I study, ${ }^{11}$ the distance from EOPYY pharmacies continued to present a major obstacle for patients, while other obstacles were related to the difficulties in obtaining the required medical diagnosis by an ESY/EOPYY physician, and to the reduced accessibility of patients to their treating physician. These barriers could be overcome through the development of an MS national registry which would standardize the distribution process (and home delivery) and assure comprehensive, and faster patient access to treatment. A registry could help EOPYY to identify, early on, the number of patients that need treatment at a given period in time, as well as the frequency of their visits to doctors and pharmacies, and therefore speed up patients' access to their medication and avoid shortages.

\section{Conclusion}

The current distribution system for high-cost medicines is a potentially effective cost-containment measure applied in the context of fiscal consolidation in Greece. Based on the findings of the present study, even though the major weaknesses of the system seem to have improved to a certain extent during the last year, there is still room for improvement.
To further ameliorate the operation of the distribution system and to remove the existing barriers in the short-term, a potential solution could be the home delivery of medicines to MS patients, especially for patients living in remote areas with poor means of transportation, tackling in this way the existing inequalities in accessing medication. Moreover, in the medium - term, the development of a national MS registry could be used to further facilitate the distribution process of high-cost medicine, ensuring patient eligibility and medicine adequacy, and thus enhancing access to care and the health status of MS patients.

\section{Acknowledgements}

We would like to thank the President of EOPYY, Mr. Dimitris Kontos, for approving this study.

\section{Ethical issues}

The President of EOPYY, Mr. Dimitris Kontos, has approved this study which was organized and implemented by the University of Peloponnese, Corinth, Greece.

\section{Competing interests}

The authors declare that they have no competing interests.

Authors' contributions

All authors contributed equally to the study and the preparation of this manuscript.

\section{Authors' affiliations}

${ }^{1}$ Faculty of Social and Political Sciences, University of Peloponnese, Corinth, Greece. ${ }^{2}$ Centre for Health Services Research, Medical School, University of Athens, Athens, Greece. ${ }^{3}$ Faculty of Social Sciences, University of Peloponnese, Corinth, Greece. ${ }^{4}$ National Organization for Healthcare Services Provision, Athens, Greece. ${ }^{5}$ Department of Statistics, Athens University of Economics and Business, Athens, Greece.

\section{References}

1. Maxwell RJ. Quality assessment in health. BrMed J (Clin Res Ed). 1984;288(6428):1470-1472. doi:10.1136/bmj.288.6428.1470

2. Põlluste $\mathrm{K}$, Kalda R, Lember $M$. Satisfaction with the access to the health services of the people with chronic conditions in Estonia. Health Policy. 2007;82(1):51-61. doi:10.1016/j. healthpol.2006.08.004

3. Põlluste K, Kallikorm R, Meiesaar K, Lember M. Satisfaction with access to health services: the perspective of Estonian patients with rheumatoid arthritis. Sci World J. 2012;2012:257569. doi:10.1100/2012/257569

4. Multiple Sclerosis International Federation. Atlas of multiple sclerosis 2013. http://www.msif.org/includes/documents/cm docs $/ 2013 / \mathrm{m} / \mathrm{msif}$-atlas-of-ms-2013-report. pdf?f=1

5. Goldenberg MM. Multiple sclerosis review. P T. 2012;37(3):175184.

6. Damal K, Stoker E, Foley JF. Optimizing therapeutics in the management of patients with multiple sclerosis: a review of drug efficacy, dosing, and mechanisms of action. Biologics. 2013;7:247-258. doi:10.2147/BTT.S53007

7. Kobelt G, Kasteng F. Access to innovative treatments in multiple sclerosis in Europe. Report prepared for the European Federation of Pharmaceutical Industry Associations (EFPIA). http://www. comparatorreports.se/Access $\% 20$ to $\% 20 \mathrm{MS} \% 20$ treatments $\% 20$ -\%200ctober\%202009.pdf. Published October 2009.

8. Wilsdon T, Barron A, Mitchell- Heggs A, Ginoza S. Access to medicines for multiple sclerosis: challenges and opportunities. Report prepared for Biogen Idec. London; February 2014. 
9. European Multiple Sclerosis Platform. MS Barometer 2013. http://www.emsp.org/wp-content/uploads/2015/06/130530-MSBarometer-2013.pdf

10. National Organization for Medicines (EOF) website. http://www. eof.gr/web/guest/protocols

11. Souliotis K, Papageorgiou M, Politi A, loakeimidis D, Sidiropoulos P. Barriers to accessing biologic treatment for rheumatoid arthritis in Greece: the unseen impact of the fiscal crisis--the Health Outcomes Patient Environment (HOPE) study. Rheumatol Int. 2014;34(1):25-33. doi:10.1007/s00296-013-2866-1

12. Laires PA, Exposto F, Mesquita R, Martins AP, Cunha-Miranda L, Fonseca JE. Patients' access to biologics in rheumatoid arthritis: a comparison between Portugal and other European countries. Eur J Health Econ. 2013;14(6):875-885. doi:10.1007/s10198-

\section{2-0432-5}

13. Putrik $\mathrm{P}$, Ramiro $\mathrm{S}$, Kvien $\mathrm{TK}$, et al. Inequities in access to biologic and synthetic DMARDs across 46 European countries. Ann Rheum Dis. 2014;73(1):198-206. doi:10.1136/ annrheumdis-2012-202603

14. Orlewska E, Ancuta I, Anic B, et al. Access to biologic treatment for rheumatoid arthritis in Central and Eastern European (CEE) countries. Med Sci Monit. 2011;17(4):SR1-SR13. doi:10.12659/ MSM.881697

15. Putrik P, Ramiro S, Kvien TK, Sokka T, Uhlig T, Boonen A. Variations in criteria regulating treatment with reimbursed biologic DMARDs across European countries. Are differences related to country's wealth? Ann Rheum Dis. 2014;73(11):20102021. doi:10.1136/annrheumdis-2013-203819 